\title{
Sensitivity and Specificity of Perfusion Scintigraphy Combined with Chest Radiography for Acute Pulmonary Embolism in PIOPED II
}

\author{
H. Dirk Sostman ${ }^{1}$, Massimo Miniati², Alexander Gottschalk ${ }^{3}$, Fadi Matta ${ }^{4}$, Paul D. Stein ${ }^{4}$, and Massimo Pistolesi² \\ ${ }^{I}$ Office of the Dean and Department of Radiology, Weill Cornell Medical College and Methodist Hospital, Houston, Texas; ${ }^{2}$ Department \\ of Critical Care, Section of Respiratory Medicine, University of Florence, Florence, Italy; ${ }^{3}$ Department of Radiology, Michigan State \\ University, East Lansing, Michigan; and ${ }^{4}$ Department of Research, St. Joseph Mercy Oakland Hospital, Pontiac, Michigan, and \\ Department of Medicine, Wayne State University, Detroit, Michigan
}

We used the archived Prospective Investigation of Pulmonary Embolism Diagnosis II (PIOPED II) data and images to test the hypothesis that reading perfusion scans with chest radiographs but without ventilation scans, and categorizing the perfusion scan as "pulmonary embolism (PE) present" or "PE absent," can result in clinically useful sensitivity and specificity in most patients. Methods: Patients recruited into PIOPED II were eligible for the present study if they had a CT angiography (CTA) or digital subtraction angiography (DSA) diagnosis, an interpretable perfusion scan and chest radiographs, and a Wells' score. Four readers reinterpreted the perfusion scans and chest radiographs of eligible patients. Two readers used the modified PIOPED II criteria and 2 used the Prospective Investigative Study of Pulmonary Embolism Diagnosis (PISAPED) criteria. The chest radiographs were read as "normal/near normal," "abnormal," or "nondiagnostic," and the perfusion scans were read as "PE present," "PE absent," or "nondiagnostic." The primary analysis used a composite reference standard: the PIOPED II DSA result or, if there was no definitive DSA result, CTA results that were concordant with the Wells' score as defined in PIOPED II (CTA positive and Wells' score $>2$, or CTA negative and Wells' score $<6)$. Results: The prevalence of PE in the sample was 169 of 889 (19\%). Using the modified PIOPED II criteria, the sensitivity of a "PE present" perfusion scan was $84.9 \%$ (95\% confidence interval [Cl], $80.1 \%-88.8 \%$ ), and the specificity of "PE absent" was $92.7 \%$ (95\% Cl, 91.1\%-94.1\%), excluding "nondiagnostic" results, which occurred in $20.6 \%(95 \% \mathrm{Cl}, 18.8 \%-22.5 \%)$. Using PISAPED criteria, the sensitivity of a "PE present" perfusion scan was $80.4 \%(95 \% \mathrm{Cl}, 75.9 \%-84.3 \%)$ and the specificity of "PE absent" was $96.6 \%$ (95\% Cl, 95.5\%-97.4\%), whereas the proportion of patients with "nondiagnostic" scans was $0 \%$ (95\% Cl, 0.0\%-0.2\%). Conclusion: Perfusion scintigraphy combined with chest radiography can provide diagnostic accuracy similar to both CTA and ventilation-perfusion scintigraphy, at lower cost and with lower radiation dose. With modified PIOPED II criteria, a higher proportion of scans were nondiagnostic than with CTA, and with PISAPED criteria none were nondiagnostic.

Received Feb. 26, 2008; revision accepted May 7, 2008.

For correspondence or reprints contact: H. Dirk Sostman, Methodist Hospital, 6565 Fannin St., Houston, TX 77030.

E-mail: dsostman@tmhs.org

COPYRIGHT @ 2008 by the Society of Nuclear Medicine, Inc.
Key Words: respiratory; vascular; PIOPED II; perfusion scintigraphy; pulmonary embolism

J Nucl Med 2008; 49:1741-1748

DOI: 10.2967/jnumed.108.052217

n many centers, CT angiography (CTA) has replaced ventilation-perfusion (V/Q) scintigraphy as the primary imaging modality for diagnosing acute pulmonary embolism (PE) (1). However, CTA is expensive and is not applicable in patients who have contraindications to iodinated contrast material. In Prospective Investigation of Pulmonary Embolism Diagnosis II (PIOPED II), 18.5\% of patients with suspected PE had elevated creatinine and $3.7 \%$ of patients were allergic to intravenous contrast material. In addition, the radiation dose from multidetector CTA recently has been identified as a major public health issue (2). Finally, recently proposed diagnostic pathways (3) are not definitive regarding further imaging options if CTA is inconclusive or discordant with the clinical probability assessment. Accordingly, additional evidence is needed on imaging modalities such as scintigraphy that could substitute for, or supplement, CTA.

The decline of V/Q use is due primarily to a high frequency of nondiagnostic readings. However, V/Q is definitive in some circumstances. A normal perfusion scan excludes PE with a negative predictive value close to $100 \%(4,5)$, and in patients with no history of prior PE, a high-probability scan has a positive predictive value greater than $90 \%$ (6). The V/Q scan has proven value, but the challenge is to increase its value by increasing the proportion of definitive results or by reducing its complexity, cost, and radiation dose.

Research on V/Q scintigraphy after the PIOPED I trial suggested that accuracy could be improved $(7,8)$ by refining diagnostic criteria and reducing the number of diagnostic categories (in particular, by combining "intermediate" and "low probability" into a "nondiagnostic" category). The sensitivity and specificity of such an approach was recently reported to be $78 \%$ and $98 \%$ (9), after excluding nondiagnostic readings that occurred in $27 \%$ of patients. Preliminary data 
suggested that the ventilation scan can be omitted without affecting diagnostic accuracy $(10,11)$. It is reasonable to suppose that this may be particularly true in patients with normal findings on chest radiography (12). Finally, the Prospective Investigative Study of Pulmonary Embolism Diagnosis (PISAPED) trial suggested a new set of diagnostic criteria to diagnose or exclude PE using the perfusion scan and chest radiograph (13) with promising accuracy and few nondiagnostic readings. If corroborated, such developments could increase the value of scintigraphy by increasing accuracy and technical simplicity while reducing cost and risk.

These considerations prompted us to evaluate further the diagnostic accuracy of perfusion scintigraphy combined with chest radiography. We report the results of reading PIOPED II perfusion scans and chest radiographs using both modified PIOPED II and PISAPED criteria to assess the accuracy of this modality for acute PE.

\section{MATERIALS AND METHODS}

\section{Patients}

The patient sample in the PIOPED II study has been described in detail (14). Patients recruited into PIOPED II were eligible for the present study if they had a CTA diagnosis or a digital subtraction angiography (DSA) diagnosis, interpretable perfusion scans and chest radiographs, and a Wells' clinical prediction rule score. All patients had given informed consent in PIOPED II for further evaluation of their study data, and no patient was personally identified in this study.

\section{Readers and Criteria}

Four experienced readers of pulmonary scintigrams independently interpreted the perfusion scans and chest radiographs. The readers had no clinical information and were not aware of the PIOPED II CTA or study classification results for any of the patients.
Two readers interpreted the perfusion scans according to the PIOPED II criteria, modified for the absence of the ventilation images (Table 1). Scan findings classified by the modified PIOPED II criteria were reported as "PE present" (high probability), "PE absent" (very low probability or normal), or "nondiagnostic" (intermediate or low probability). Two other readers interpreted the same scans according to the PISAPED criteria (Table 1) as "PE present," "PE absent," or "nondiagnostic."

The PIOPED readers characterized the chest radiography findings as "normal/near normal" if there was no parenchymal opacity apart from a few small $(<1 \mathrm{~cm})$ nodules or diffuse lung disease with low profusion and low density; no bullae; no pleural effusion or opacity greater than the costophrenic sulcus; and no extrapulmonary (e.g., cardiac or mediastinal contour) abnormality large enough to obscure most of the lungs. Oligemia was not considered an abnormality. If the chest radiographs showed other positive radiographic findings, they were classified as "abnormal."

In examining the chest radiographs, the PISAPED readers considered the size and shape of the heart and hilar arteries, position of the diaphragm, presence or absence of pulmonary parenchymal abnormalities (consolidation, atelectasis, oligemia, edema), and pleural effusion. For the hilar arteries, attention was paid to the presence of abrupt vascular amputation that gives the hilum a "plump" appearance (15). Pulmonary consolidations were considered suggestive of infarction if they had a semicircular or half-spindle shape and were arranged peripherally along the pleural surface (15). Oligemia was considered to be present if, in a given lung region, the pulmonary vasculature was greatly diminished with or without concomitant hyperlucency of the lung parenchyma (15). Chest radiographs were rated as abnormal if one or more of the following were present: enlargement of the heart or hilar vessels; elevated diaphragm (unilateral or bilateral); pleural effusion (including intrafissural liquid); increased lung density (focal or diffuse); pulmonary edema; oligemia with or without pleonemia in the contralateral lung; consolidation suggestive of infarction; emphysema; or fibrothorax.

If the quality of the images was poor, the chest radiographs were rated as "nondiagnostic" by both groups of readers according to

TABLE 1

Modified PIOPED II and PISAPED Scintigraphic Criteria

\begin{tabular}{|c|c|c|}
\hline \multirow[b]{2}{*}{ Finding } & \multicolumn{2}{|l|}{ Criteria } \\
\hline & Modified PIOPED II & PISAPED \\
\hline PE present & High probability (2 or more segments of perfusion-chest radiograph mismatch) & $\begin{array}{l}\text { One or more wedge-shaped } \\
\text { perfusion defects }\end{array}$ \\
\hline \multirow[t]{8}{*}{ PE absent } & Normal perfusion & Normal perfusion \\
\hline & Very low probability & \\
\hline & $\begin{array}{l}\text { Nonsegmental lesion, for example, prominent hilum, cardiomegaly, elevated } \\
\text { diaphragm, linear atelectasis, or costophrenic angle effusion with no other } \\
\text { perfusion defect in either lung radiographic lesion }\end{array}$ & Near normal \\
\hline & Perfusion defect smaller than radiographic lesion & $\begin{array}{l}\text { Contour defect caused by } \\
\text { enlarged heart, mediastinum, } \\
\text { or diaphragm }\end{array}$ \\
\hline & $1-3$ small segmental defects & $\begin{array}{l}\text { Perfusion defect, not } \\
\text { wedge-shaped }\end{array}$ \\
\hline & $\begin{array}{l}\text { Solitary chest radiograph-perfusion matched defect in mid or upper lung } \\
\text { zone confined to single segment }\end{array}$ & \\
\hline & Stripe sign around perfusion defect (best tangential view) & \\
\hline & $\begin{array}{l}\text { Pleural effusion in at least one third of pleural cavity, with no other perfusion } \\
\text { defect in either lung }\end{array}$ & \\
\hline Not diagnostic & All other findings & $\begin{array}{l}\text { Cannot classify as PE-positive } \\
\text { or PE-negative }\end{array}$ \\
\hline
\end{tabular}


the reader's subjective impression of whether the images were evaluable for the purposes of the study.

\section{Data Recording}

Each reader completed an electronic form directly on a personal computer, recording the reader's identity, the patient's identification code, the chest radiography category, the scan category, and comments.

\section{Data Analysis}

The primary analysis compared the present study perfusion scan results with the PIOPED II V/Q scan results (9). We computed the sensitivity and specificity of the perfusion scan using the same diagnostic reference standard adopted in a recent analysis of the sensitivity and specificity of the V/Q scan (9). The reference standard was the PIOPED II DSA result, if available. If DSA was not done or was not definitive, the reference standard was the PIOPED II CTA results that were concordant with the Wells' score (16) (i.e., CTA positive and Wells' score $>2$, or CTA negative and Wells' score < 6), which (in a comparable sample in PIOPED II) resulted in positive and negative predictive values of $93 \%$ (14). Patients with discordance between CTA and Wells' score were excluded from the analysis. Sensitivity, specificity, and confidence intervals (CIs) were computed with an online calculator (http:// www.causascientia.org/math_stat/ProportionCI.html) for each reader and also for pooled modified PIOPED II and PISAPED readings. We excluded "nondiagnostic" scintigram readings from the computation of sensitivity and specificity (17), as was done for computing CTA sensitivity and specificity in PIOPED II and for a recent analysis of V/Q scan accuracy $(9,14)$. We computed interobserver variability for modified PIOPED II and PISAPED scan readings using the $\kappa$-statistic (18).

We performed 2 prespecified subgroup analyses. We compared the sensitivity and specificity of perfusion scan readings in patients who had normal or nearly normal chest radiography findings with the sensitivity and specificity of perfusion scan readings in patients with abnormal or nondiagnostic chest radiographs and in patients younger than or older than $50 \mathrm{y}$ of age.

Finally, we compared the concordance of perfusion scan readings with the PIOPED II CTA consensus central reading. We used the $\kappa$-statistic as an index of concordance between CTA and perfusion scan results.

\section{RESULTS}

The PIOPED II patient sample contained 1,062 records with a CTA diagnosis. Of these, 37 were excluded for lack of a perfusion scan and 115 for failure to meet the criteria for the reference diagnosis. The usable PIOPED II sample thus consisted of 910 cases. Of these, 21 were later eliminated because of incomplete images in the PIOPED II archives, discovered during the reading process (Fig. 1).

The prevalence of PE in the sample was 169 of 889 (19\%). The reference diagnosis was established by DSA in 210 cases and by CTA plus Wells' score in the remaining 679 .

Table 2 delineates the sensitivity and specificity for each reader, as well as the proportion of nondiagnostic readings. The modified PIOPED II readings and PISAPED readings showed no statistically significant difference in sensitivity and specificity. The modified PIOPED II readings had sig- nificantly more nondiagnostic readings $(20.6 \%)$ than did the PISAPED readings $(0 \%)$. Interobserver agreement was moderate between the 2 PIOPED readers $(\kappa=0.537)$ and almost perfect between the 2 PISAPED readers $(\kappa=0.903)$. A summary comparison of the perfusion scan readings with the PIOPED II V/Q scan readings is shown in Table 3. The results of both the modified PIOPED II and the PISAPED perfusion scan readings were similar to the PIOPED II V/Q scan results that were reported previously (9). Table 4 shows the predictive values for modified PIOPED II and PISAPED readings and compares them with the PIOPED II data for CTA (14).

In the subgroup analysis of patients with normal or abnormal chest radiography findings, the PIOPED readers rated $72 \%$ of chest radiographs as showing normal or near-normal findings, whereas the PISAPED readers rated $77 \%$ of chest radiographs as showing abnormal findings. From $0.4 \%$ to $2.8 \%$ of chest radiographs were rated as nondiagnostic by different readers. There were no nondiagnostic scans for PISAPED readings in patients with normal or near-normal chest radiography findings or patients with abnormal or nondiagnostic chest radiography findings, but for the modified PIOPED II readings, there were far more nondiagnostic scans (45.4\%; 95\% CI, 41.0\%-50.0\%) in the abnormal subgroup than in the normal or near-normal subgroup $(10.9 \%$; $95 \% \mathrm{CI}$, 9.4\%-12.8\%). For the modified PIOPED II readings, the sensitivity of the perfusion scan when the chest radiography findings were normal was $86.3 \%$ (95\% CI, $81.0 \%-90.4 \%$ ), and specificity was $93.1 \%$ (95\% CI, $91.3 \%-94.6 \%)$; when the chest radiography findings were abnormal, the sensitivity was $78.4 \%$ (95\% CI, $65.3 \%-87.5 \%$ ) and specificity was $90.8 \%$ (95\% CI, 86.0\%-94.0\%). For the PISAPED readings, the sensitivity of the perfusion scan when the chest radiography findings were normal was $72.3 \%$ (95\% CI, 58.2\%-83.0\%) and specificity was $96.8 \%$ (95\% CI, $94.3 \%-98.2 \%)$; when the chest radiography findings were abnormal, the sensitivity was $81.9 \%$ (95\% CI, 76.9\%-86.0\%) and specificity was $96.5 \%$ (95\% CI, 95.3\%-97.5\%).

In the subgroup analysis of patients under $50 \mathrm{y}$ old and patients $50 \mathrm{y}$ old or older, we did not find a strong or consistent trend in perfusion scan accuracy as a function of age. The results are shown in Table 5. The PIOPED readers, but not the PISAPED readers, categorized scans as "nondiagnostic" more frequently in patients aged $50 \mathrm{y}$ or older than in patients younger than 50 y (25.9\% vs. $14.7 \%$ nondiagnostic).

The crude agreement between CTA and perfusion scanning was $89.7 \%$ and $93.7 \%$ for the 2 PIOPED readings and $93.0 \%$ and $92.8 \%$ for the 2 PISAPED readings. The $\kappa$-statistic of agreement between CTA and perfusion scanning was 0.693 and 0.799 for the 2 PIOPED readings and 0.765 and 0.774 for the 2 PISAPED readings, indicating "substantial" agreement (18).

\section{DISCUSSION}

The role of CTA in clinical practice rests on a foundation of solid evidence. A prospective, multicenter accuracy 


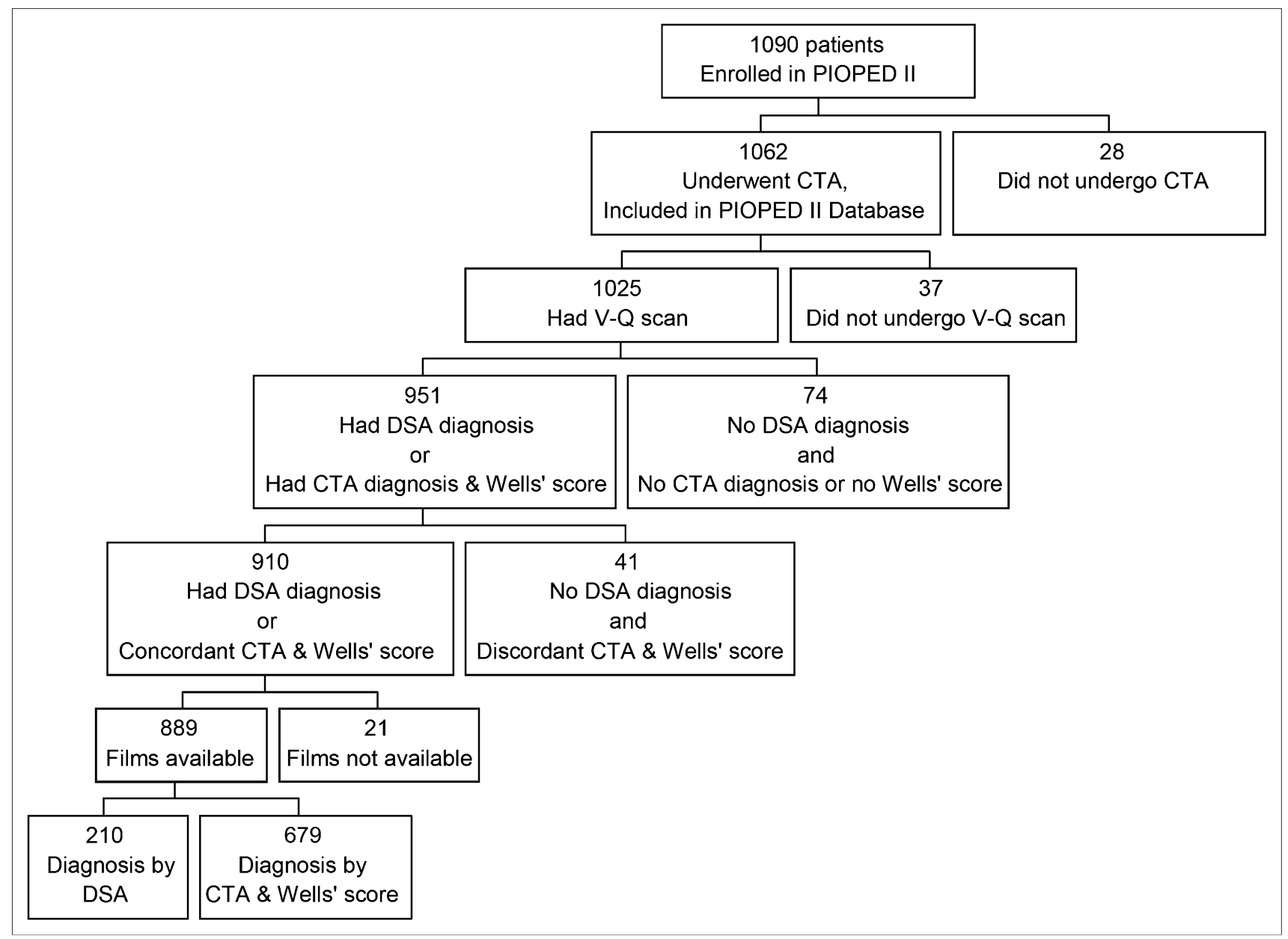

FIGURE 1. Selection of patients from those enrolled in PIOPED II was based on presence of defined diagnostic reference standard and availability of interpretable scintigraphic images. Of 1,090 patients enrolled in PIOPED II, 180 were not eligible for inclusion in our analysis: 28 did not undergo CTA, 37 did not undergo V/Q scanning, 74 of those who did not have a DSA diagnosis either did not have a CTA diagnosis or did not have a Wells' score, and in another 41 of those who did not have a DSA diagnosis, CTA diagnosis and Wells' score were discordant. Discordance between CTA and Wells' score was defined according to PIOPED II (14) criteria: low clinical probability (Wells' score $<2$ ) with CTA positive for PE, or high clinical probability (Wells' score $>6$ ) with CTA negative for PE. Finally, in 21 cases that met selection criteria, films could not be located for rereading. In this flow chart, both CTA and DSA were of pulmonary arteries.

study (14) established that CTA, when used in combination with objective clinical assessment, has sensitivity and specificity sufficiently high for clinical management. Outcome studies $(19,20)$ have shown that the diagnosis of PE can safely be excluded by negative results on multislice CTA. However, CTA is expensive and may lead to complications in patients who have reduced renal function or iodine allergy. In addition, recently proposed diagnostic pathways (3) may require further imaging, such as scintigraphy, if CTA is inconclusive or discordant with the clinical probability assessment. Finally, the increasingly frequent use of CTA has raised concern about the radiation exposure of the population $(2,21,22)$ and, in particular, radiation of the female breast. The breast dose with CTA in an average 60-kg woman is $20 \mathrm{mGy} /$ breast (23), $50-80 \mathrm{mSv}$ based on coronary CTA (24), and as much as 190 mGy with CTA in a woman with large breasts (25). Because of the risk of cancer after such exposures to radiation, an imaging test with lower doses may be preferred, especially in younger women. Breast irradiation with V/Q scintigraphy is approximately $0.28-0.9 \mathrm{mGy}(26)$, which is less than $5 \%$ of the radiation dose to the breast resulting from CTA. This characteristic of scintigraphy would be even more important if follow-up studies are needed to evaluate restoration of pulmonary perfusion over time, or in patients who may have recurrent clinical episodes of suspected PE.

However, V/Q use has declined, compared with CTA, primarily because of a high frequency of nondiagnostic readings. In PIOPED I (32\% outpatients), V/Q scans gave a definitive diagnosis in only $28 \%$ of patients (6). More recently, in a mixed population of inpatients and outpatients, $46 \%$ had a definitive diagnosis by V/Q scanning (27). However, V/Q has long been accepted as definitive in some circumstances. A normal perfusion scan excludes PE 
TABLE 2

Diagnostic Results

\begin{tabular}{|c|c|c|c|c|}
\hline \multirow[b]{2}{*}{ Index } & \multicolumn{2}{|c|}{ Modified PIOPED II } & \multicolumn{2}{|c|}{ PISAPED } \\
\hline & Reader 1 & Reader 2 & Reader 1 & Reader 2 \\
\hline Sensitivity & $84.9 \%(107 / 126)$ & $85.0 \%(113 / 133)$ & $81.7 \%(138 / 169)$ & $79.3 \%(134 / 169)$ \\
\hline $95 \% \mathrm{Cl}$ & $77.6 \%-90.1 \%$ & $77.9 \%-90.0 \%$ & $75.1 \%-86.7 \%$ & $72.5 \%-84.7 \%$ \\
\hline Specificity & $89.4 \%(512 / 573)$ & $96.0 \%(557 / 580)$ & $96.7 \%(696 / 720)$ & $96.5 \%(695 / 720)$ \\
\hline $95 \% \mathrm{Cl}$ & $86.6 \%-91.6 \%$ & $94.1 \%-97.3 \%$ & $95.1 \%-97.7 \%$ & $94.9 \%-97.6 \%$ \\
\hline Nondiagnostic readings & $21 \%(190 / 889)$ & $20 \%(176 / 889)$ & $0 \%(0 / 889)$ & $0 \%(0 / 889)$ \\
\hline
\end{tabular}

with a negative predictive value close to $100 \%(4,5)$, whereas a high-probability scan has a positive predictive value of $88 \%$ and, in patients without a history of prior PE, 91\% (6). Accordingly, the V/Q scan has proven value, but the challenge is to increase its value by increasing the proportion of definitive results or by reducing the cost, radiation dose, and time required for testing by eliminating the ventilation scan.

Research on scintigraphy since the PIOPED I trial has suggested that the accuracy of interpretation could be improved $(7,8)$ by refining diagnostic criteria and reducing the number of diagnostic categories. A very-low-probability interpretation of the V/Q scan was found to be as reliable as CTA in excluding PE when the Wells' clinical prediction rule indicated either a low or a moderate probability of PE (8). Retrospective analysis of data from PIOPED II $(75 \%$ outpatients) showed a definitive V/Q scan result in $74 \%$ of patients, with sensitivity of $78 \%$ and specificity of $98 \%$, after exclusion of the nondiagnostic scans (9). In some populations, scintigraphy may result in over $90 \%$ of definitive diagnoses (28). Finally, a recent randomized trial showed equivalent outcomes for patients evaluated with a clinical prediction rule who were randomized to either CTA or V/Q scintigraphy, although more cases of PE were detected by CTA (27). Accordingly, although an enormous amount of past investigation has been done regarding scintigraphy, further improvements in its clinical value appear to be achievable and important.

A noteworthy change since the PIOPED I trial has been reduction in "nondiagnostic" readings; PIOPED I had
$76.6 \%$ nondiagnostic scans (32.6\% low-probability and $44.0 \%$ intermediate-probability), whereas PIOPED II had only $26.5 \%$ (9.8\% low-probability and $16.7 \%$ intermediateprobability). Elimination of the ventilation scan reduces both cost and radiation exposure (21). The ventilation scan can be eliminated in many patients without reducing diagnostic accuracy $(4,5,11,13)$. However, a more systematic and complete evaluation of the perfusion scan-only approach was needed to assess sensitivity and specificity rigorously and to evaluate whether eliminating the ventilation scan affects the proportion of "nondiagnostic" readings. Our data show that in the PIOPED II population, eliminating the ventilation scan does not reduce overall accuracy. If outcome studies confirm the safety of this approach, perfusion scintigraphy could be highly beneficial for patients in whom CTA may not be preferred. The highest priority for outcome analysis of perfusion-only scintigraphy should be in young women, since the risks of radiation are higher in women $(2,24)$ and in younger individuals $(2,24)$. Of course, in clinical practice it is universally accepted that all patients should be spared needless exposure to radiation and financial cost, irrespective of age and sex.

The main goal of the present study was to evaluate the sensitivity, specificity, and rate of nondiagnostic perfusion scans read with chest radiographs. We wished to determine whether it is possible to increase the value of scintigraphy by, first, providing definite interpretations with reasonable accuracy and, second, eliminating routine use of the ventilation scan to reduce the cost, complexity, and radiation

TABLE 3

V/Q Scan Results Compared with Perfusion Scan Results

\begin{tabular}{|c|c|c|c|}
\hline \multirow[b]{2}{*}{ Index } & \multirow[b]{2}{*}{ PIOPED II V/Q Scan* } & \multicolumn{2}{|c|}{ Perfusion Scan ${ }^{\dagger}$} \\
\hline & & Modified PIOPED II & PISAPED \\
\hline Sensitivity & $77.4 \%(89 / 115)$ & $84.9 \%(220 / 259)$ & $80.5 \%(272 / 338)$ \\
\hline $95 \% \mathrm{Cl}$ & $69.7 \%-85.0 \%$ & $80.1 \%-88.8 \%$ & $75.9 \%-84.3 \%$ \\
\hline Specificity & $97.7 \%(541 / 554)$ & $92.7 \%(1,069 / 1,153)$ & $96.6 \%(1,391 / 1,440)$ \\
\hline $95 \% \mathrm{Cl}$ & $96.4 \%-98.9 \%$ & $91.1 \%-94.1 \%$ & $95.5 \%-97.4 \%$ \\
\hline Nondiagnostic readings & $26.5 \%(241 / 910)$ & $20.6 \%(366 / 1,778)$ & $0.0 \%(0 / 1,778)$ \\
\hline $95 \% \mathrm{Cl}$ & $23.6 \%-29.3 \%$ & $18.8 \%-22.5 \%$ & $0.0 \%-0.2 \%$ \\
\hline $\begin{array}{l}{ }^{*} \text { Data from Sostman et } \\
{ }^{\dagger} \text { Pooled data for } 2 \text { reade }\end{array}$ & & & \\
\hline
\end{tabular}


TABLE 4

Predictive Values of Perfusion Scan Compared with CTA

\begin{tabular}{lccc}
\hline \multicolumn{1}{c}{ Test } & PPV & NPV & Not diagnostic \\
\hline Modified PIOPED perfusion scan* & $72.4 \%(220 / 304)$ & $96.5 \%(1,069 / 1,108)$ & $20.6 \%(366 / 1,778)$ \\
PISAPED perfusion scan* & $84.7 \%(272 / 321)$ & $95.5 \%(1,391 / 1,457)$ & $0.0 \%(0 / 1,778)$ \\
CTA $^{\dagger}$ & $85.7 \%(150 / 175)$ & $94.8 \%(567 / 598)$ & $6.2 \%(51 / 824)$ \\
& & & \\
\hline & & & \\
${ }^{*}$ Pooled data for 2 readers. & & & \\
${ }^{\dagger}$ Data from Stein et al., Table 4 (14). & & & \\
\hline
\end{tabular}

dose of scintigraphy. In addition, we wished to evaluate different criteria for interpreting perfusion scans and the accuracy of perfusion scans in patients with normal or abnormal chest radiography findings and in younger or older patients.

The PIOPED II composite reference standard was not used in this study, because the V/Q scan was often a definitive or influential component of it and, thus, incorporation bias would have been present. We chose to use the DSA result when available, since DSA is the most widely accepted imaging reference standard. If no DSA result was available, we used the CTA result if it was concordant with the Wells' score. The V/Q scan had some influence on selection of patients to undergo DSA in PIOPED II and thus affected the spectrum of patients who had a diagnosis by DSA, but the PIOPED II DSA readings were independent of other imaging or clinical information. The CTA readings and Wells' score determinations also were independent observations. We chose to use only those CTA results that were concordant with the Wells' scores, since the PIOPED II study showed that CTA-Wells' concordance was associated with high positive and negative predictive values (14).

In a separate analysis, we also evaluated the concordance between the perfusion scan findings and CTA diagnoses, since one of the clinical issues is whether perfusion scanning can be substituted for CTA. The high concordance indicated that this is the case, consistent with the results from a recent outcome study of V/Q scans and CTA (27).

TABLE 5

Perfusion Scan Results According to Patient Age*

\begin{tabular}{rll}
\hline \multicolumn{1}{c}{ Index } & Modified PIOPED II & \multicolumn{1}{c}{ PISAPED } \\
\hline Age $<50$ y & & \\
Sensitivity & $79.1 \%(87 / 110)$ & $83.3 \%(110 / 132)$ \\
$95 \% \mathrm{Cl}$ & $70.5 \%-85.6 \%$ & $76.0 \%-88.7 \%$ \\
Specificity & $94.7 \%(573 / 605)$ & $97.0 \%(685 / 706)$ \\
$95 \% \mathrm{Cl}$ & $92.6 \%-96.2 \%$ & $95.5 \%-98.0 \%$ \\
Age $\geq 50$ y & & \\
Sensitivity & $89.3 \%(133 / 149)$ & $78.6 \%(162 / 206)$ \\
$95 \% \mathrm{Cl}$ & $83.2 \%-93.2 \%$ & $72.5 \%-83.7 \%$ \\
Specificity & $90.5 \%(496 / 548)$ & $96.2 \%(706 / 734)$ \\
$95 \% \mathrm{Cl}$ & $(87.8 \%-92.7 \%)$ & $(94.5 \%-97.3 \%)$
\end{tabular}

*Pooled data for 2 readers.
The results of the PISAPED and modified PIOPED II readings have both similarities to and differences from CTA and each other. When modified PIOPED II criteria were used, there were more nondiagnostic results with perfusion scans $(20.6 \%$ of patients) than there were with CTA in PIOPED II (6.2\% of patients). Further, virtually all nondiagnostic CTA results were due to technically inadequate scans (28), whereas the nondiagnostic perfusion scan results were primarily due to inconclusive interpretations. Once nondiagnostic studies were removed from the study sample, the sensitivity and specificity of perfusion scans read using the modified PIOPED II criteria (85\% and 93\%, pooled data from the 2 readings) were similar to those reported in PIOPED II for CTA (83\% and 96\%). When the PISAPED criteria were used, there were fewer nondiagnostic results with perfusion scans ( $0 \%$ of patients) than there were with CTA in PIOPED II (6.2\% of patients), yet sensitivity and specificity were still similar to CTA $(80 \%$ and $97 \%$, pooled data from the 2 readings). The PISAPED readers also had higher interobserver agreement than the PIOPED readers, possibly because of the use of the "nondiagnostic" category in the modified PIOPED II readings, or the fact that the 2 PISAPED readers worked together daily in the same institution, or the greater simplicity of the PISAPED criteria. An additional factor influencing the results may be the level of experience of the PIOPED readers with perfusion-only images; one PIOPED reader had been an interpreter in the PISAPED study (13) and thus had more experience using scintigraphy without ventilation images than did the other PIOPED reader, who had no prior experience.

As in PIOPED I (12), the data did not indicate a consistent relationship between normal findings on chest radiographs and more accurate perfusion scans. The accuracy of the modified PIOPED II readings was slightly higher when the chest radiography findings were normal or nearly normal; however, the proportion of nondiagnostic scans was much lower for normal chest radiography findings than when the findings were abnormal. Accordingly, if one uses the modified PIOPED II criteria, the perfusion scan could be expected to be more clinically useful in patients with normal or near-normal chest radiography findings. With the PISAPED readings, there was no difference in accuracy between the normal and abnormal subgroups. Comparison between the modified PIOPED II and PISAPED results for chest radiograph subgroups is confounded by the differences in 
criteria for normal and abnormal chest radiography findings. It may well be that the chest radiography interpretation has a significant effect on perfusion scan accuracy. The modified PIOPED II chest radiograph classification was quite simple and was primarily used as a ventilation scan surrogate, whereas the PISAPED chest radiograph classification was more sophisticated and likely to introduce additional useful diagnostic information. However, harvesting such information likely requires skill and experience with chest radiography interpretation at a level that many readers may not possess. In addition, the data do not indicate a clear relationship between younger age and more accurate perfusion scans. The PIOPED readers, but not the PISAPED readers, more frequently rated the scans of patients aged $50 \mathrm{y}$ or older as "nondiagnostic"; hence, the trends for modified PIOPED II and PISAPED readings were inconsistent. Accordingly, young age and normal chest radiography findings can predict that the perfusion scan will give more clinically useful results with the modified PIOPED II criteria, because of fewer nondiagnostic readings, but accuracy did not vary with age or chest radiography findings, and the nondiagnostic rate of the PISAPED readings did not vary.

Our study has limitations. That DSA was not done on all patients is a limitation, albeit one that all future studies of PE will share because of the vanishing use of invasive pulmonary angiography in clinical practice. The use of concordant CTA and Wells' score as an alternate diagnostic reference standard has not been prospectively validated in an independent study. However, in the PIOPED II study (14), the concordant CTA and Wells' score had positive and negative predictive values of $93 \%$ (predictive value is the relevant statistic when one is considering a reference standard test in a population with comparable composition). It has even been suggested that CTA may be a better reference standard than DSA (29). Furthermore in the PISAPED study (13), perfusion scans were compared with an angiographic reference standard in all patients, and the sensitivity and specificity were comparable to those determined in the present study.

Another limitation relates to imaging technique. First, the V/Q scans in PIOPED II often used aerosols to image ventilation, and in several cases activity from the ventilation images was present on the perfusion scans, potentially confounding the interpretation (either by obscuring perfusion defects or, alternatively, by providing some information about regional ventilation). Second, regardless of the use of ventilation images, the PIOPED II scintigrams were planar, and (in principle) better results might be obtained with tomographic perfusion imaging. Finally, the majority of the PIOPED II patients were outpatients, in contrast to the population of the PIOPED I study; thus, we cannot be sure our results would apply to a more severely ill population of inpatients. However, the PISAPED study (13) did have a majority of inpatients, with a $39 \%$ prevalence of $\mathrm{PE}$, and the PISAPED study also included rereading by a single interpreter of 723 perfusion scans from the PIOPED I study, with a perfusion scan sensitivity of $80 \%$ and specificity of $83 \%$ (13). Accordingly, we would not anticipate substantial degradation of diagnostic results in a more severely ill population. Of course, the prevalence of PE in the population will affect the predictive values of the scan for any given sensitivity and specificity.

Our study has implications regarding the role of scintigraphy in patients with suspected acute PE. The advantages of CTA - rapid and widely available examinations, the ability to perform lower-extremity venous imaging concurrently if needed, the high proportion of definitive diagnostic results, and the ability of CT to provide additional diagnostic information $(30,31)$ that may be of clinical importance-secure a place for CTA as a first-line diagnostic imaging modality in appropriately selected patients. However, the advantages of perfusion scintigraphy-lower cost, much lower radiation dose, avoidance of iodinated contrast material, and technical simplicity-can now be considered together with a contemporary characterization of the diagnostic accuracy of the test in a predominantly outpatient population. Increased use of scintigraphy appears warranted in centers that recently have relegated it to a minor supporting role (32).

As to whether the results of this study support the current clinical use of the PISAPED criteria rather than the modified PIOPED criteria, our data do not suffice to resolve this issue definitively. With the readers in this study, PISAPED had comparable sensitivity and specificity to the modified PIOPED II while greatly reducing the number of nondiagnostic scans. However, it remains to be shown that readers without extensive experience using the PISAPED methodology could achieve these results. Clinical care should be guided by local expertise and results.

\section{CONCLUSION}

We conclude that perfusion scintigraphy is accurate and usually diagnostically definitive. It can be considered the pulmonary imaging procedure of choice for the potentially large number of patients in whom CTA may be disadvantageous. The principal difference between modified PIOPED II and PISAPED readings is more nondiagnostic results with the modified PIOPED II criteria, making the PISAPED criteria more clinically useful in experienced hands. Those who apply the modified PIOPED II criteria may find them more useful in younger patients with nearly normal chest radiography findings. The results of perfusion scanning are similar to those of V/Q scanning and of CTA. Ventilation scans are superfluous in most patients.

\section{ACKNOWLEDGMENT}

This study was supported by grants HL063981 and HL067453 from the U.S. Department of Health and Human Services, Public Health Service, National Heart, Lung, and Blood Institute, Bethesda, Maryland. 


\section{REFERENCES}

1. Stein PD, Kayali F, Olson RE. Trends in the use of diagnostic imaging in patients hospitalized with acute pulmonary embolism. Am J Cardiol. 2004;93:1316-1317.

2. Brenner DJ, Hall EJ. Computed tomography: an increasing source of radiation exposure. N Engl J Med. 2007;357:2277-2284.

3. Stein PD, Woodard PK, Weg JG, et al. Diagnostic pathways in acute pulmonary embolism: recommendations of the PIOPED II investigators. Am J Med. 2006; 119:1048-1055.

4. Kipper MS, Moser KM, Kortman KE, et al. Long-term follow-up in patients with suspected pulmonary embolism and a normal lung scan. Chest. 1982;82:411-415.

5. Hull RD, Raskob GE, Coates G, et al. Clinical validity of a normal perfusion lung scan in patients with suspected pulmonary embolism. Chest. 1990;97:23-26.

6. Value of the ventilation/perfusion scan in acute pulmonary embolism: results of the prospective investigation of pulmonary embolism diagnosis (PIOPED). The PIOPED Investigators. JAMA. 1990;263:2753-2759.

7. Sostman HD, Coleman RE, Newman GE, DeLong D, Paine SS. Evaluation of revised criteria for ventilation-perfusion scintigraphy in patients with suspected pulmonary embolism. Radiology. 1994;193:103-107.

8. Gottschalk A, Stein PD, Sostman HD, et al. Very low probability interpretation of ventilation-perfusion lung scans in combination with low probability clinical assessment reliably excludes pulmonary embolism: data from PIOPED II. $\mathrm{J} \mathrm{Nucl}$ Med. 2007;48:1411-1415.

9. Sostman HD, Stein PD, Gottschalk A, et al. Sensitivity and specificity of ventilation-perfusion scintigraphy for acute pulmonary embolism in PIOPED II. Radiology. 2008;246:941-946.

10. Goldberg SN, Palmer EL, Scott JA, et al. Pulmonary embolism: prediction of the usefulness of initial ventilation-perfusion scanning with chest radiographic findings. Radiology. 1994;193:801-805.

11. Stein PD, Terrin ML, Gottschalk A, et al. Value of ventilation/perfusion scans compared to perfusion scans alone in acute pulmonary embolism. Am J Cardiol. 1992;69:1239-1241.

12. Stein PD, Alavi A, Gottschalk A, et al. Usefulness of non-invasive diagnostic tools for diagnosis of acute pulmonary embolism in patients with a normal chest radiograph. Am J Cardiol. 1991;67:1117-1120.

13. Miniati M, Pistolesi M, Marini C, et al. Value of the perfusion lung scan in the diagnosis of pulmonary embolism. Am J Respir Crit Care Med. 1996;154:13871393.

14. Stein PD, Fowler SE, Goodman LR, et al. Multidetector computed tomography for acute pulmonary embolism. N Engl J Med. 2006;354:2317-2327.

15. Miniati M, Monti S, Bottai M. A structured clinical model for predicting the probability of pulmonary embolism. Am J Med. 2003;114:173-179.
16. Wells PS, Anderson DR, Rodger M, et al. Excluding PE at the bedside without diagnostic imaging: management of patients with suspected PE presenting to the emergency department by using a simple clinical model and D-dimer. Ann Intern Med. 2001;135:98-107.

17. Simel DL, Feussner JR, Delong ER, Matchar DB. Intermediate, indeterminate and uninterpretable diagnostic tests. Med Decis Making. 1987;7:107-114.

18. Landis JR, Koch GG. The measurement of observer agreement for categorical data. Biometrics. 1977;33:159-174.

19. Perrier A, Roy P-M, Sanchez O, et al. Multidetector-row computed tomography in suspected pulmonary embolism. N Engl J Med. 2005;352:1760-1768.

20. The Christopher Study Investigators. Effectiveness of managing suspected pulmonary embolism using an algorithm combining clinical probability, D-dimer testing, and computed tomography. JAMA. 2006;295:172-179.

21. Remy-Jardin M, Pistolesi M, Goodman LR, et al. Management of suspected acute pulmonary embolism in the era of CT angiography: a statement of the Fleischner Society. Radiology. 2007;245:315-329.

22. Amis ES Jr, Butler PF, Applegate KE, et al. American College of Radiology white paper on radiation dose in medicine. J Am Coll Radiol. 2007;4:272-284.

23. Parker MS, Hui FK, Camacho MA. Female breast radiation exposure during CT pulmonary angiography. AJR. 2005;185:1228-1233.

24. Einstein AJ, Henzlova MJ, Rajagopalan S. Estimating risk of cancer associated with radiation exposure from 64-slice computed tomography coronary angiography. JAMA. 2007;298:317-323.

25. Milne ENC. Female breast radiation exposure [letter]. AJR. 2006;186:E24.

26. Radiation dose to patients from radiopharmaceuticals (addendum 2 to ICRP publication 53). Ann ICRP. 1998;28:1-126.

27. Anderson DR, Kahn SR, Rodger MA, et al. Computerized tomographic pulmonary angiography versus ventilation-perfusion lung scanning in patients with suspected pulmonary embolism: a randomized controlled trial. JAMA. 2007;298: 2743-2753.

28. Forbes KP, Reid JH, Murchison JT. Do preliminary chest X-ray findings define the optimum role of pulmonary scintigraphy in suspected pulmonary embolism? Clin Radiol. 2001;56:397-400.

29. Jones SE, Wittram C. The indeterminate CT pulmonary angiogram: imaging characteristics and patient clinical outcome. Radiology. 2005;237:329-337.

30. Coche EE, Muller NL, Kim K-I, Wiggs BR, Mayo JR. Acute pulmonary embolism: ancillary findings at spiral CT. Radiology. 1998;207:753-758.

31. Shah AA, Davis SD, Gamsu G, Intriere L. Parenchymal and pleural findings in patients with and patients without acute pulmonary embolism detected at spiral CT. Radiology. 1999;211:147-153.

32. Freeman LM. Don't bury the V/Q scan: it's as good as multidetector CT angiograms with a lot less radiation exposure. J Nucl Med. 2008;49:5-8. 